Proyecciones Journal of Mathematics

Vol. 34, $\mathrm{N}^{o}$ 1, pp. 45-54, March 2015.

Universidad Católica del Norte

Antofagasta - Chile

\title{
The multi-step homotopy analysis method for solving the Jaulent-Miodek equations
}

\author{
Mohammad Zurigat \\ Al Al-Bayt University, Jordan \\ Asad A. Freihat \\ Al-Balqa Applied University, Jordan \\ and \\ Ali H. Handam \\ Al Al-Bayt University, Jordan \\ Received : October 2014. Accepted : December 2014
}

\begin{abstract}
In this work, the multi-step homotopy analysis method (MHAM) is applied to obtain the explicit analytical solutions for system of the Jaulent Miodek equations. The proposed scheme is only a simple modification of the homotopy analysis method (HAM), in which it is treated as an algorithm in a sequence of small intervals (i.e. time step) for finding accurate approximate solutions to the corresponding problems. Thus, it is valid for both weakly and strongly nonlinear problems. this work verifies the validity and the potential of the MHAM for the study of nonlinear systems. A comparative study between the new algorithm and the exact solution is presented graphically. convenient.
\end{abstract}

Subjclass [2000] : 11 Y35, 65L05.

Keywords : Differential algebraic equations; multi-step homotopy analysis method; Numerical solutions. 


\section{Introduction}

Recently, a lot of attention has been focused on the studies of linear and nonlinear systems of partial differential equations (PDEs). Systems of nonlinear partial differential equations arise in many scientific models such as the propagation of shallow water waves and the Brusselator model of the chemical reaction-diffusion model. In this work we study the JaulentMiodek equations $[1,12,16]$ which associate with energy-dependent Schrödinger potential $[2,13,15]$. There are many methods to solve this system, such as F-function method [3], Adomian method [4], homotopy analysis [12, 16], tanh method [5], and the variational iteration method [6]. In this paper, we propose the multi-step homotopy analysis method to solve the JaulentMiodek equations. We investigate the applicability and effectiveness of the homotopy analysis method when treated as an algorithm in a sequence of intervals (i.e. time step) for finding accurate approximate solutions to the Jaulent-Miodek equations. It can be found that the corresponding numerical solutions obtained by using HAM are valid only for a short time, while the ones obtained by using MHAM are more valid and accurate during a long time [10]. The structure of this paper is as follows. In section 2, we describe the MHAM of the system of nonlinear Jaulent-Miodek equations. Numerical simulations are presented graphically in Section 3. Finally, the conclusions are given in Section 4.

\section{Multi-step homotopy analysis method}

The principles of the homotopy analysis method are given in $[7,8,9,10,11]$. The nonlinear Jaulent-Miodek equations which will be considered in this paper has the following form:

$$
\mathrm{u}_{t}+u_{x x x}+\frac{3}{2} v v_{x x x}+\frac{9}{2} v_{x} v_{x x}-6 u u_{x}-6 u v v_{x}-\frac{3}{2} v^{2} u_{x}=0,
$$

$$
v_{t}+v_{x x x}-6 v u_{x}-6 u v_{x}-\frac{15}{2} v^{2} v_{x}=0,
$$

with initial conditions

$$
u(x, 0)=\frac{1}{8} \lambda^{2}\left(1+4 \operatorname{sech}^{2}\left[\frac{1}{2} \lambda x\right]\right), \quad v(x, 0)=\lambda \operatorname{sech}\left[\frac{1}{2} \lambda x\right],
$$

which associate with energy-dependent Schrödinger potential [13, 14, 15]. The HAM is used to provide approximate solutions for a wide class of nonlinear problems in terms of convergent series with easily computable components, but the motivation of this article is to extend the HAM to 
solve system of the Jaulent-Miodek (JM) equations. this modification is called the multi-step homotopy analysis method (MHAM). It is only a simple modification of the standard HAM and can ensure the validity of the approximate solutions for large time. Although the MHAM is used to provide approximate solutions for nonlinear problem in terms of convergent series with easily computable components. To extend this solution over the interval $[0, t]$, we divide the interval $[0, t]$ into $n$-subintervals of equal length $\Delta t,\left[t_{0}, t_{1}\right),\left[t_{1}, t_{2}\right),\left[t_{2}, t_{3}\right), \ldots,\left[t_{n-1}, t_{n}\right]$ with $t_{0}=0, t_{n}=t$. Let $t^{*}$ be the initial value for each subintervals and let $u_{j}$ and $v_{j}$ be approximate solutions in each subinterval $\left[t_{j-1}, t_{j}\right], j=1,2, \ldots, n$, with initial guesses

$u_{1}\left(x, t^{*}\right)=\frac{1}{8} \lambda^{2}\left(1+4 \operatorname{sech}^{2}\left[\frac{1}{2} \lambda x\right]\right), \quad u_{j}\left(x, t^{*}\right)=U_{j}\left(x, t_{j-1}\right)=U_{j-1}\left(x, t_{j-1}\right)$,

$v_{1}\left(x, t^{*}\right)=\lambda \operatorname{sech}\left[\frac{1}{2} \lambda x\right], v_{j}\left(x, t^{*}\right)=V_{j}\left(x, t_{j-1}\right)=V_{j-1}\left(x, t_{j-1}\right), \quad j=2,3, \ldots, n$.

Now, we can construct the so-called zeroth-order deformation equations of the system (2.1) by

$$
\begin{aligned}
& (1-q) L\left[\phi_{1, j}(x, t, q)-u_{j}\left(x, t^{*}\right)\right]=q h\left[\frac{\partial}{\partial t} \phi_{1, j}(x, t, q)+\frac{\partial^{3}}{\partial x^{3}} \phi_{1, j}(x, t, q)\right. \\
& +\frac{3}{2} \phi_{2, j}(x, t, q) \frac{\partial^{3}}{\partial x^{3}} \phi_{2, j}(x, t, q)+\frac{9}{2} \frac{\partial}{\partial x} \phi_{2, j}(x, t, q) \frac{\partial^{2}}{\partial x^{2}} \phi_{2, j}(x, t, q) \\
& -6 \phi_{1, j}(x, t, q) \frac{\partial}{\partial x} \phi_{1, j}(x, t, q)-6 \phi_{1, j}(x, t, q) \phi_{2, j}(x, t, q) \frac{\partial}{\partial x} \phi_{2, j}(x, t, q) \\
& -\frac{3}{2}\left(\phi_{2, j}(x, t, q)\right)^{2} \frac{\partial}{\partial x} \phi_{1, j}(x, t, q), \\
& (1-q) L\left[\phi_{2, j}(x, t, q)-v_{j}\left(x, t^{*}\right)\right]=q h\left[\frac{\partial}{\partial t} \phi_{2, j}(x, t, q)+\frac{\partial^{3}}{\partial x^{3}} \phi_{2, j}(x, t, q)\right.
\end{aligned}
$$

$$
\begin{aligned}
& -6 \phi_{2, j}(x, t, q) \frac{\partial}{\partial x} \phi_{1, j}(x, t, q)-6 \phi_{1, j}(x, t, q) \frac{\partial}{\partial x} \phi_{2, j}(x, t, q) \\
& -\frac{15}{2}\left(\phi_{2, j}(x, t, q)\right)^{2} \frac{\partial}{\partial x} \phi_{2, j}(x, t, q),
\end{aligned}
$$

where $q \in[0,1]$ is an embedding parameter, $L$ is an auxiliary linear operator, $h \neq 0$ is an auxiliary parameter and $\phi_{1, j}(x, t, q)$ and $\phi_{2, j}(x, t, q)$ are unknown functions. Obviously, when $q=0$ 


$$
\begin{gathered}
\phi_{1,1}(x, t, 0)=\frac{1}{8} \lambda^{2}\left(1+4 \operatorname{sech}^{2}\left[\frac{1}{2} \lambda x\right]\right), \quad \phi_{1, j}(x, t, 0)=U_{j-1}\left(x, t_{j-1}\right), \\
\phi_{2,1}(x, t, 0)=\lambda \operatorname{sech}\left[\frac{1}{2} \lambda x\right], \quad \phi_{2, j}(x, t, 0)=V_{j-1}\left(x, t_{j-1}\right), \quad j=2,3, \ldots, n,
\end{gathered}
$$

and when $q=1$, we have

$$
\begin{gathered}
\phi_{1, j}(x, t, 1)=u_{j}(x, t), \\
\phi_{2, j}(x, t, 1)=v_{j}(x, t), \quad j=1,2, \ldots, n .
\end{gathered}
$$

Expanding $\phi_{1, j}(x, t, q)$ and $\phi_{2, j}(x, t, q), j=1,2, \ldots, n$, in Taylor series with respect to $q$, we get

$$
\begin{gathered}
\phi_{1, j}(x, t, q)=u_{j}\left(x, t^{*}\right)+\sum_{m=1}^{\infty} u_{j, m}(x, t) q^{m} \\
\phi_{2, j}(x, t, q)=v_{j}\left(x, t^{*}\right)+\sum_{m=1}^{\infty} v_{j, m}(x, t) q^{m}, \quad j=1,2, \ldots, n,
\end{gathered}
$$

where

$$
\begin{aligned}
& u_{j, m}(x, t)=\left.\frac{1}{m !} \frac{\partial^{m} \phi_{1, j}(x, t, q)}{\partial q^{m}}\right|_{q=0}, \\
& v_{j, m}(x, t)=\left.\frac{1}{m !} \frac{\partial^{m} \phi_{2, j}(x, t, q)}{\partial q^{m}}\right|_{q=0} .
\end{aligned}
$$

If the initial guesses $u_{j}\left(x, t^{*}\right), v_{j}\left(x, t^{*}\right)$, the auxiliary linear operator $L$ and the nonzero auxiliary parameter $h$ are properly chosen so that the power series (2.7) converges at $q=1$, one has

$$
\begin{aligned}
& u_{j}(x, t)=\phi_{1, j}(x, t, 1)=u_{j}\left(x, t^{*}\right)+\sum_{m=1}^{\infty} u_{j, m}(x, t), \\
& v_{j}(x, t)=\phi_{2, j}(x, t, 1)=v_{j}\left(x, t^{*}\right)+\sum_{m=1}^{\infty} v_{j, m}(x, t) .
\end{aligned}
$$

Define the vectors

$$
\begin{aligned}
& \vec{u}_{j, m}(x, t)=\left\{u_{j, 0}(x, t), u_{j, 1}(x, t), \ldots, u_{j, m}(x, t)\right\}, \\
& \vec{v}_{j, m}(x, t)=\left\{v_{j, 0}(x, t), v_{j, 1}(x, t), \ldots, v_{j, m}(x, t)\right\} .
\end{aligned}
$$

Differentiating the zero-order deformation equation (2.4) $m$ times with respective to $q$, then setting $q=0$ and dividing them by $m$ !, finally using (2.8), we have the so-called high-order deformation equations 


$$
\begin{aligned}
& L\left[u_{j, m}(x, t)-\chi_{m} u_{j, m-1}(x, t)\right]=h \Re_{1, j, m}\left(\vec{u}_{j, m-1}(x, t)\right), \\
& L\left[v_{j, m}(x, t)-\chi_{m} v_{j, m-1}(x, t)\right]=h \Re_{2, j, m}\left(\vec{v}_{j, m-1}(x, t)\right),
\end{aligned}
$$

subject to the initial conditions

$$
u_{j, m}(x, 0)=0, \quad v_{j, m}(x, 0)=0, j=1,2, \ldots, n,
$$

where

$$
\begin{aligned}
& \Re_{1, j, m}\left(\vec{u}_{j, m-1}(x, t)\right) \\
& =\frac{\partial}{\partial t} u_{j, m-1}(x, t)+\frac{\partial^{3}}{\partial x^{3}} u_{j, m-1}(x, t)+\frac{3}{2} \sum_{i=0}^{m-1} v_{j, i}(x, t) \frac{\partial^{3}}{\partial x^{3}} v_{j, m-i-1}(x, t) \\
& +\frac{9}{2} \sum_{i=0}^{m-1} \frac{\partial}{\partial x} v_{j, i}(x, t) \frac{\partial^{2}}{\partial x^{2}} v_{j, m-i-1}(x, t)-6 \sum_{i=0}^{m-1} u_{j, i}(x, t) \frac{\partial}{\partial x} u_{j, m-i-1}(x, t) \\
& -6 \sum_{i=0}^{m-1} \frac{\partial}{\partial x} v_{j, m-i-1}(x, t) \sum_{k=0}^{i} u_{j, k}(x, t) v_{j, i-k}(x, t) \\
& -\frac{3}{2} \sum_{i=0}^{m-1} \frac{\partial}{\partial x} u_{j, m-i-1}(x, t) \sum_{k=0}^{i} v_{j, k}(x, t) v_{j, i-k}(x, t), \\
& \Re_{2, j, m}\left(\vec{v}_{j, m-1}(x, t)\right) \\
& =\frac{\partial}{\partial t} v_{j, m-1}(x, t)+\frac{\partial^{3}}{\partial x^{3}} v_{j, m-1}(x, t)-6 \sum_{i=0}^{m-1} v_{j, i}(x, t) \frac{\partial}{\partial x} u_{j, m-i-1}(x, t) \\
& -6 \sum_{i=0}^{m-1} \frac{\partial}{\partial x} v_{j, i}(x, t) u_{j, m-i-1}(x, t) \\
& -\frac{15}{2} \sum_{i=0}^{m-1} \frac{\partial}{\partial x} v_{j, m-i-1}(x, t) \sum_{k=0}^{i} v_{j, k}(x, t) v_{j, i-k}(x, t), \\
& \underbrace{}_{i=0}{ }^{m},
\end{aligned}
$$

and

$$
\chi_{m}=\left\{\begin{array}{cc}
0, & m \leq 1 \\
1, & m>1
\end{array} .\right.
$$

Select the auxiliary linear operator $L=\frac{\partial}{\partial t}$, then the mth-order deformation equations (2.9) can be written in the form

$$
u_{j, m}(x, t)=\chi_{m} u_{j, m-1}(x, t)+h \int_{0}^{t} \Re_{1, j, m}\left(\vec{u}_{j, m-1}(x, \tau)\right) d \tau,
$$




$$
v_{j, m}(x, t)=\chi_{m} v_{j, m-1}(x, t)+h \int_{0}^{t} \Re_{2, j, m}\left(\vec{v}_{j, m-1}(x, \tau)\right) d \tau, \quad j=1,2, \ldots, n .
$$

The solutions of system $(2.1)$ in each subinterval $\left[t_{j-1}, t_{j}\right], j=1,2, \ldots, n$, has the form

$$
\begin{gathered}
U_{j}(x, t)=\sum_{m=0}^{\infty} u_{j, m}\left(x, t-t_{j-1}\right), \\
V_{j}(x, t)=\sum_{m=0}^{\infty} v_{j, m}\left(x, t-t_{j-1}\right), \quad j=1,2, \ldots, n,
\end{gathered}
$$

and the solution of system $(2.1)$ for $[0, T]$ is given by

$$
u(x, t)=\sum_{j=1}^{n} \chi_{v} U_{j}(x, t), \quad v(x, t)=\sum_{j=1}^{n} \chi_{v} V_{j}(x, t),
$$

where

$$
\chi_{v}= \begin{cases}1, & t \in\left[t_{j-1}, t_{j}\right] \\ 0, & t \notin\left[t_{j-1}, t_{j}\right]\end{cases}
$$

\section{Numerical results}

The series solutions of the functions $u(x, t)$ and $v(x, t)$ are given in (2.12). In this work, we carefully propose the MHAM, a reliable modification of the HAM, that improves the convergence of the series solution. Furthermore, we should note that if one chooses a good enough initial guess, one can get accurate approximations using MHAM by only a few terms with $h=-1$.To demonstrate the efficiency of the MHAM for Jaulent-Miodek equations, we compare approximate solutions of $\mathrm{u}(\mathrm{x}, \mathrm{t})$ and $\mathrm{v}(\mathrm{x}, \mathrm{t})$, with exact solutions

$$
\begin{gathered}
u(x, t)=\frac{1}{8} \lambda^{2}\left(1+4 \sec h^{2}\left[\frac{1}{2} \lambda\left(x+\frac{1}{2} \lambda^{2} t\right)\right]\right), \\
v(x, t)=\lambda \sec h\left[\frac{1}{2} \lambda\left(x+\frac{1}{2} \lambda^{2} t\right)\right],
\end{gathered}
$$

where $\lambda$ is arbitrary constant. To demonstrate the effectiveness of the proposed algorithm as an approximate tool for solving the Jaulent-Miodek equations (2.1), (2.2) for larger $t$, we apply the proposed algorithm on the interval $[0,30]$. We choose to divide the interval $[0,30]$ to subintervals. 
Figure 1 shows the series solution of the MHAM of the Jaulent-Miodek equations (2.1), (2.2) and the displacement of the exact solution (3.1) when $(\lambda=0.1)$. From the graphical results it can be seen that the results obtained using the MHAM match the results of the exact solution very well. Therefore, the proposed method is very effcient and accurate method that can be used to provide analytical solutions for linear and nonlinear partial differential equations..

\section{Conclusions}

The analytical approximations to the solutions of Jaulent-Miodek equations (2.1), (2.2) are reliable and confirm the power and ability of the MHAM as an easy device for computing the solution of nonlinear problems. In this paper, a system of partial differential equations is studied and its approximate solution is presented using a MHAM. The approximate solutions obtained by MHAM are highly accurate and valid for a long time. The reliability of the method and the reduction in the size of computational domain give this method a wider applicability. Finally, the recent appearance of nonlinear partial differential equations as models in some fields such as models in science and engineering makes it is necessary to investigate the method of solutions for such equations, and we hope that this work is a step in this direction.

\section{References}

[1] M. Jaulent and J. Miodek, Nonlinear evolution equations associated with energy-dependent Schrödinger potentials, Lett Math Phys 1, pp. 243-250, (1976).

[2] J. L. Zhang, M. L. Wang, and X. R. Li, The subsidiary ellipticlike equation and the exact solutions of the higher-order nonlinear Schrödinger equation, Chaos Solitons Fractals, 33, pp. 1450-1457, (2007).

[3] E. Fan, Uniformly constructing a series of explicit exact solutions to nonlinear equations in mathematical physics, Chaos Solitons Fractals, 16, pp. 819-839, (2003). 
[4] J. B. Chen and X. G. Geng, Decomposition to the modified JaulentMiodek hierarchy, Chaos Solitons Fractals, 30, pp. 797-803, (2006).

[5] A. M. Wazwaz, The tanh-coth and the sech methods for exact solutions of the Jaulent-Miodek equation, Phys Lett A, 366, pp. 85-90, (2007).

[6] D. D. Ganji, M. Jannatabadi, and E. Mohseni, Application of He's variational iteration method to nonlinear Jaulent-Miodek equations and comparing it with ADM, J Comput Appl Math, 207, pp. 35-45, (2007).

[7] J. Cang, Y. Tan, H. Xu, S. Liao, Series solutions of non-linear Riccati differential equations with fractional order, Chaos, Solitons \& Fractals, 40 (1), pp. 1-9, (2009).

[8] M. Zurigat, S. Momani, Z. odibat, A. Alawneh, The homotopy analysis method for handling systems of fractional differential equations, Applied Mathematical Modelling, 34 (1), pp. 24-35, (2010).

[9] M. Zurigat, S. Momani, A. Alawneh, Analytical approximate solutions of systems of fractional algebraic-differential equations by homotopy analysis method, Computers and Mathematics with Applications, 59 (3), pp. 1227-1235, (2010).

[10] A. K. Alomari, M. S. M. Noorani, R. Nazar, C. P. Li, Homotopy analysis method for solving fractional Lorenz system, Commun Nonliear Sci Numer Simult, 15 (7), pp. 1864-1872, (2010).

[11] A. Rafiq, M. Rafiullah, Some multi-step iterative methods for solving nonlinear equations, Computers \& Mathematics with Applications, 58 (8), pp. 1589-1597, (2009).

[12] M. M. Rashidi, G. Domairry and S. Dinarvand, The Homotopy Analysis Method for Explicit Analytical Solutions of Jaulent-Miodek Equations, Numerical Methods for Partial Differential Equations, 25 (2), pp. 430-439, (2008).

[13] H. T. Ozer and S. Salihoglu, Nonlinear Schrödinger equations and N = 1 superconformal algebra, Chaos Solitons Fractals 33, pp. 1417-1423, (2007).

[14] J. L. Zhang, M. L. Wang, and X. R. Li, The subsidiary ellipticlike equation and the exact solutions of the higher-order nonlinear 
Schrödinger equation, Chaos Solitons Fractals 33, pp. 1450-1457, (2007).

[15] J. M. Zhu and Z. Y. Ma, Exact solutions for the cubic-quintic nonlinear Schrödinger equation, Chaos Solitons Fractals 33, pp. 958-964, (2007)

[16] A. Yildirim and A. Kelleci, Numerical Simulation of the Jaulentmiodek Equation byHe's Homotopy Perturbation Method,World Applied Sciences Journal 7 (Special Issue for Applied Math), (2009).
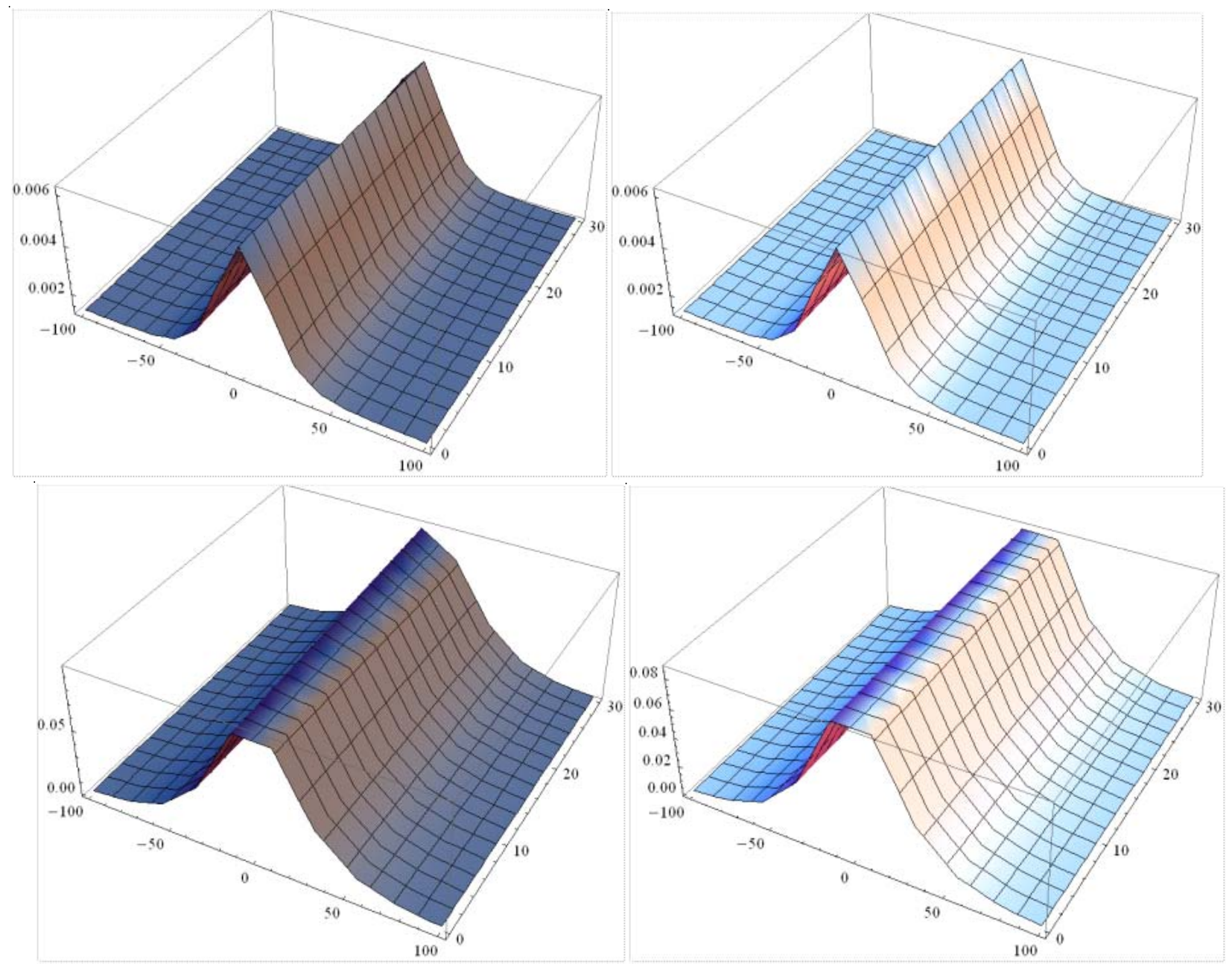

Fig. 1. The surfaces show exact and approximate solutions of $\mathrm{u}(\mathrm{x}, \mathrm{t})$ and $\mathrm{v}(\mathrm{x}, \mathrm{t})$
(a) $\mathrm{u}_{M H A M}$,
(b) $\mathrm{u}_{e x}$
(c) $\mathrm{v}_{M H A M}$,
(d) $\mathrm{v}_{e x}$. 


\section{Zurigat}

Department of Mathematics,

Al al-Bayt University

P. O. Box: 130095,

Al Mafraq,

Jordan

e-mail : moh_zur@hotmail.com

Asad A. Freihat

Department of Applied Science

Ajloun College

Al-Balqa Applied University

Ajloun 281

Jordan

e-mail : asadfreihat@yahoo.com

and

\section{Ali H. Handam}

Department of Mathematics,

Al al-Bayt University

P. O. Box: 130095,

Al Mafraq,

Jordan

e-mail : ali.handam@windowslive.com 